Research paper

\title{
Effect of plasticity on dynamic impact in a journal-bearing system: A planar case
}

\author{
Q. Peng ${ }^{\mathrm{a}}, \mathrm{X} . \mathrm{Ye}^{\mathrm{a}}, \mathrm{H} . \mathrm{Wu}^{\mathrm{b}, \mathrm{c}}, \mathrm{X} . \mathrm{Liu}^{\mathrm{a}, \mathrm{c}, *}$, Y.G. Wei ${ }^{\mathrm{d}}$ \\ a State Key Laboratory of Nonlinear Mechanics, Institute of Mechanics, Chinese Academy of Sciences, Beijing 100190, China \\ ${ }^{\mathrm{b}}$ Key Laboratory for Mechanics in Fluid Solid Coupling Systems, Institute of Mechanics, Chinese Academy of Sciences, Beijing 100190, \\ China \\ ' School of Engineering Science, University of Chinese Academy of Sciences, Beijing 100049, PR China \\ d College of Engineering, Peking University, Beijing 100871, China
}

\section{A R T I C L E I N F O}

\section{Article history:}

Received 16 June 2020

Revised 12 July 2020

Accepted 20 July 2020

Available online 31 July 2020

\section{Keywords:}

Conformal contact

Dynamic impact

Normal force-displacement relationship

Plastic deformation

Revolute joint

\begin{abstract}
A B S T R A C T
Plasticity is inevitable during contact with a high load, and thus should be considered when simulating high-load contact. Plasticity during non-conformal contact has been well studied. However, no contact law that considers plasticity during conformal contact has been developed, even though such "plastic conformal contact" commonly occurs in journal-bearing systems under high loads. Herein, we constructed a plastic Winkle model of plastic conformal contact, and integrated it into a contact solver to simulate the impact dynamics of a single journal-bearing system. The plastic contact law was first verified by finite-element simulations, which were in good agreement with the plastic indentation observed during loading and unloading. To study the plasticity during conformal impact, we integrated the contact law into a dynamic-contact model of the single journal-bearing system. The results indicate that the energy dissipation due to plasticity was much greater than that from damping and friction. Ignoring plasticity may result in an unrealistic travel distance of the journal during impact. The developed model was used to construct a velocity map to determine whether or not that plasticity should be considered.
\end{abstract}

(c) 2020 Elsevier Ltd. All rights reserved.

\section{Introduction}

Clearance is necessary in a linkage joint; the small gap between the connected bodies can constrain translation and ensure component assembly, yet also permits rotation. Most mechanical systems, including some mobile overconstrained mechanisms, are connected by revolute joints, also known as journal bearings, where small clearances are always present $[1,2]$. Such clearances for mechanical connections, commonly referred to as 'backlash', may have a large effect on vibration, noise, and wear during operation [3]. For example, Erkaya showed that clearance-induced vibration can make dynamics of system worse [4]. Thus, understanding the dynamics of a journal-bearing system according to the small clearance will shed light on the design of complex mechanical transmission systems, which normally contain many joint linkages.

This topic has been studied extensively. Theoretical and experimental approaches to the dynamics of clearance joints have been reported by Haines [5], Flores [6], Liu and Yu [7], and Wang and Liu [8]. These investigations mainly focused on modeling motion in contact according to clearance, and the relative suitability of friction and hysteresis models. Considering

\footnotetext{
* Corresponding author at: State Key Laboratory of Nonlinear Mechanics, Institute of Mechanics, Chinese Academy of Sciences, Beijing 100190, China.

E-mail address: xiaomingliu@imech.ac.cn (X. Liu).
} 


\author{
List of Symbols \\ $C_{i} i \quad$ th contact point \\ $c_{\mathrm{e}} \quad$ Restitution coefficient \\ D Damping coefficient \\ $E^{*} \quad$ Equivalent elastic modules \\ $\boldsymbol{e}_{i n} \quad$ Unit normal vector of the $i$ th collision \\ $\boldsymbol{e}_{i \mathrm{t}} \quad$ Unit tangent vector of the $i$ th collision \\ $f \quad$ Friction \\ $K_{0} \quad$ Indentation stiffness \\ $k \quad$ Regression coefficient \\ $L_{i} \quad$ Travel distance of the journal between the $i$ th and $(i+1)$ th collision \\ $m \quad$ Journal mass \\ $J \quad$ Moment of inertia of the journal \\ $P \quad$ Contact force \\ $R_{\mathrm{b}} \quad$ Bearing radius \\ $R_{\mathrm{j}} \quad$ Journal radius \\ $\Delta R \quad$ Radius difference: $R_{\mathrm{b}}-R_{\mathrm{j}}$ \\ $\boldsymbol{r}_{0} \quad$ Initial position of the journal \\ $V_{\text {cr }} \quad$ Critical speed \\ $\boldsymbol{V}_{0} \quad$ Initial velocity of the journal \\ $\boldsymbol{V}_{i} \quad$ Velocity of the journal's mass center at the $i$ th collision \\ $\boldsymbol{V}_{\text {in }} \quad$ Normal velocity of the journal's mass center at the $i$ th collision \\ $\boldsymbol{V}_{i t} \quad$ Tangent velocity of the journal's mass center at the $i$ th collision \\ $\boldsymbol{V}_{i \mathrm{cn}} \quad$ Normal approaching velocity of the contact point at the $i$ th collision \\ $\boldsymbol{V}_{i c t} \quad$ Tangent approaching velocity of the contact point at the $i$ th collision \\ $\boldsymbol{V}_{i \mathrm{icn}} \quad$ Normal departure velocity of the contact point at the $i$ th collision \\ $\boldsymbol{V}^{\prime}{ }_{i c t} \quad$ Tangent departure velocity of the contact point at the $i$ th collision \\ $\alpha_{\mathrm{a} i} \quad$ Approaching angle of the $i$ th collision \\ $\alpha_{\mathrm{d} i} \quad$ Departure angle of the $i$ th collision \\ $\delta \quad$ Compressive displacement \\ $\delta_{\mathrm{cr}} \quad$ Critical compressive displacement \\ $\sigma_{\mathrm{Y}} \quad$ Yield stress \\ $\omega_{i} \quad$ Angular speed of the journal after the $i$ th collision \\ $\omega_{\text {ref }} \quad$ Reference angular speed \\ $\mu \quad$ Hysteresis damping factor \\ $\mu_{f} \quad$ Coefficient of friction
}

these factors is essential for realistic modeling or prediction of joint system dynamics. Recently, Tian, Flores, and Lankarani comprehensively reviewed the numerical and experimental methodologies used to evaluate the dynamics of multibody mechanical systems having journal-bearing clearance [9]. They concluded that most of the previous contact models for journal bearings are elastic. To model cylindrical joints with clearances, two approaches have generally been taken [10]: the constraint approach [11] and the contact force approach [12-14]. The former approach improves the analysis of dynamics via additional geometrical constraints, while the latter is more realistic since it considers impacts between objects.

To model a journal-bearing system via the contact force approach, the contact model is very important for precise simulation results. Hertz contact theory has also been applied in an attempt to formulate the problem of the clearance of a journal bearing, and to study the dynamic response [15,16]. The compressive displacement must be small [10], because this model is limited to non-conformal contact.

The solution of two-dimensional (2D) conformal contact, which has been widely used to model journal bearings, was reported by Persson [17]. Although he provided a closed form solution to normal force-displacement (NFD) in conformal contact, the solution was limited to the case of elastic similarity. Ciavarella and Decuzzi [18,19] presented a closed-form relationship between the normal load and contact angle in the cases of elastic similarity and dissimilarity. The NFD relationship was derived using a complex integral calculation and was not explicit, thus adding complexity to dynamic simulations.

Johnson [20], Goldsmith [21], Lankarani-Nikravesh [22], and Radzimovsky [23] have reported NFD models that resemble the Hertz model. However, these models are approximations that only agree with other conformal solutions, such as Persson [17] and Ciavarella and Decuzzi [18,19], over a small compressive displacement range. Limitations of the models have been noted; for example, Pereira et al. concluded that the models of Goldsmith and Lankrani-Nikravesh are inappropriate for modeling low-clearance conformal contact between cylinders [24,25]. However, their enhanced model, based on the contact model of Johnson [26,27], is suitable for low contact loads. 


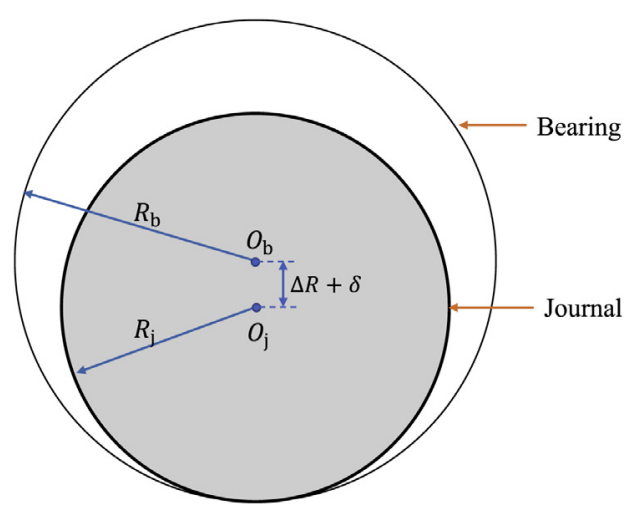

Fig. 1. Schematic diagram of a cylindrical joint with clearance.

Several numerical methods have been proposed to derive a more accurate NFD relationship for journal bearings. Liu et al. $[10,28]$ introduced a model for approximating the NFD relationship based on Winkler elastic foundation model. In their study, they presumed a distribution of contract pressure with a peak value proportional to the compressive displacement. The model was established by determining the proportional coefficient via finite element simulation. This approach works very well for an elastic case with small load. However, finite element simulations and theoretical calculations using their Hertzian-type stress distribution model were not in good agreement with actual behavior under heavy loads. Fang et al. proposed a universal solution for normal contact between frictionless spherical surfaces by combining analytical and numerical methods [29]. However, their solution is too complex to be integrated into a dynamic code.

Skrinjar et al. [30] and Wang and Liu [31] reviewed various contact-force models that are commonly used in the study of multibody dynamics. The models considered force in two parts; the first part is proportional to the compressive displacement to the power of 1.5 , and the other part is related to the time derivative of the compressive displacement. By modifying these models, Zhang et al. used nondimensional analysis to develop a new continuous contact force model to accommodate complex geometries and energy dissipations in multibody system [32]. However, due to the former part of the force, such models are only suitable for small dynamic loads. For high contact loads, plasticity must be taken into account. Many plastic contact models have been developed for non-conformal and sphere-to-sphere contact. However, very few studies have considered the effect of plastic on conformal contact. For modeling plastic non-conformal contact, the models of Jackson and Green [33] and Kogut and Etsion [34] are suitable when a deformable hemisphere is in contact with a rigid flat surface; the models of Kogut and Komvopoulos [35], Ye and Komvopoulos [36], Brake [37], Stronge [38], and Thornton [39], and a modified version of the Jackson-Green model by Ghaednia et al. [40] are suitable when a rigid hemisphere is in contact with a deformable half-space. For plastic conformal contact, our previous work proposed a solution for the NFD relationship of elastoplastic conformal contact. Plasticity was considered in our plastic Winkler model to ensure that it is capable of dealing with realistic engineering problems such as high loads and plasticity [41].

We investigated the effect of plasticity on the dynamics of the clearance joint as follows. We used a normal contact model [41] considering plasticity and a tangential contact model considering Coulomb friction to study the motions of a journalbearing pair. Generally, in a real-world situation, clearance may introduce mechanistic defects, such as misalignment. Studies on misalignment [42-44] revealed that it introduces local complexity into the system, such as friction in a 3D space [42], contact nonlinearity [43], or a complicated contact detection algorithm. In the present study, an explicit force-displacement model accounting for the effects of plasticity was validated by finite element model (FEM) simulations and implemented into a 2D dynamics solver. In the solver, once collision is detected, functions for solving the impact process are called. Based on a typical planar dynamic system, i.e., a journal/bearing pair, we investigated the effects of plasticity on joint dynamics. The paper is organized as follows. First, in Section 2, an NFD relationship is verified. A damping effect is added to the model to take account of energy loss other than that from plastic dissipation. Then, a planar dynamical model of a revolute joint with clearance is developed, in which continuous contact force and friction are considered. In Section 3, by comparing the results simulated by elasto-damping $(\mathrm{E}+\mathrm{D})$ and elasto-plasto-damping $(\mathrm{E}+\mathrm{P}+\mathrm{D})$ models, we determine the effect of plastic deformation on journal motion. It is shown that for some cases involving large impact velocity, plasticity must be considered for a more accurate prediction. This paper emphasizes the effects of plasticity and provides a new method to solve the dynamic process with considering plasticity.

\section{Formulation}

\subsection{Contact model}

Conformal contact between two cylindrical bodies is illustrated in Fig. 1. The geometric and material parameters are listed in the notation. According to our previous model [41], the NFD relationship for elastoplastic conformal contact can be 


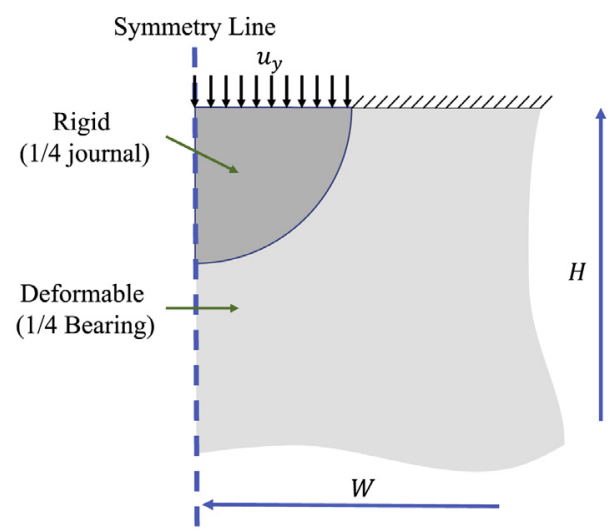

Fig. 2. FEM Model for cylindrical joints with clearance. Due to symmetry, only a quarter of the joint is modeled. The journal is set to be rigid and the bearing to is deformable.

expressed as follows:

$$
P= \begin{cases}\left(k E^{*}\left(\frac{2}{3} \delta^{\frac{3}{2}}-g^{\frac{1}{2}} \delta+\frac{1}{3} g^{\frac{3}{2}}\right)+R_{\mathrm{b}} \sigma_{\mathrm{Y}} g^{\frac{1}{2}}\right) \sqrt{\frac{8}{\Delta R+\delta}} & , \text { if } g \geq 0 \text { and } \mathrm{d} \delta>0 \\ \frac{8}{3} k E^{*} \delta \sqrt{\frac{\delta}{2(\Delta R+\delta)}} & , \text { if } g<0 \text { and } \mathrm{d} \delta>0 \\ \frac{8}{3} k E^{*}\left(\delta-\delta_{p}\right) \sqrt{\frac{\delta-\delta_{p}}{2\left(\Delta R+\delta-\delta_{p}\right)}} & , \text { if } \mathrm{d} \delta \leq 0\end{cases}
$$

where $\delta$ is the compressive displacement or penetration displacement, $\delta_{p}$ is the permanent displacement caused by plastic deformation, $E^{*}$ is the equivalent modulus, $\sigma_{\mathrm{Y}}$ is the yielding stress, $R_{\mathrm{b}}$ is the bearing radius, $\Delta R$ is the difference between the bearing radius and journal radius, $k$ is a constant used to fit the model to the FEM simulation, and

$$
g=\delta-\frac{\sigma_{\mathrm{Y}} R_{\mathrm{b}}}{k E^{*}}
$$

Eq. (2) identifies the criterion for establishing that plastic deformation has taken place. The critical value then can be expressed as follows:

$$
\delta_{\mathrm{cr}}=\frac{\sigma_{\mathrm{Y}} R_{\mathrm{b}}}{k E^{*}}
$$

The compressive displacement during contact can be nondimensionalized by $\delta_{\mathrm{cr}}$, as follows:

$$
\bar{\delta}=\frac{\delta}{\delta_{\mathrm{cr}}}
$$

and the normal contact force by:

$$
\bar{P}=\frac{P}{\sigma_{\mathrm{Y}} R_{\mathrm{b}} \cdot B}
$$

where $B=1$ refers to the unit length off plane.

The NFD relationship for elastoplastic conformal contact in Eq. (1) is compared with the two commonly used models (i.e., Johnson's and Radzimovsky's) and the Finite Element Method (FEM) simulation. FEM simulations of a cylindrical joint with small clearance were carried out using ABAQUS commercial software (Dassualt Systèmes, Vélizy-Villacoublay, France). A static solving procedure (ABAQUS/Standard) was used to derive the force-displacement relationship of a conformal indentation, in which a rigid journal and a deformable bearing are modeled with $\Delta R / R_{b}=0.001$. The equivalent modulus $E^{*}$ is set at $100 \mathrm{GPa}$, and the ratio of $\sigma_{\mathrm{Y}}$ to $E^{*}$ is 0.001 . Plane stress elements (CPS3) were adopted because a journal bearing in operation is more akin to a plane-stress situation rather than a plane-strain one. A contact pair is established between the rigid journal and the deformable bearing: the penalty method of constraint enforcement is applied on the normal direction for the pressure-overclosure relationship ('Hard' contact in ABAQUS), while the tangential direction is set to be frictionless.

Due to the symmetry of cylindrical joints, only a quarter of the joint was modeled, shown in Fig. 2. The symmetrical boundary is shown as the dashed line. The top surface of the outer body is fixed, and the inner journal is displaced with fixed displacement $u_{y}$ in the vertical direction from the top. The dimensions of the outer body in the horizontal direction and vertical direction (defined as $W$ and $H$, respectively) are set to be greater than $500 R_{\mathrm{b}}$ to eliminate the boundary effect. The bottom and right side of the outer body are set as free. The mesh in the FEM model, shown in Fig. 3, is applied with a suitable element size in the contact area between the cylinder and outer body. The mesh size in the cylinder has a gradient 


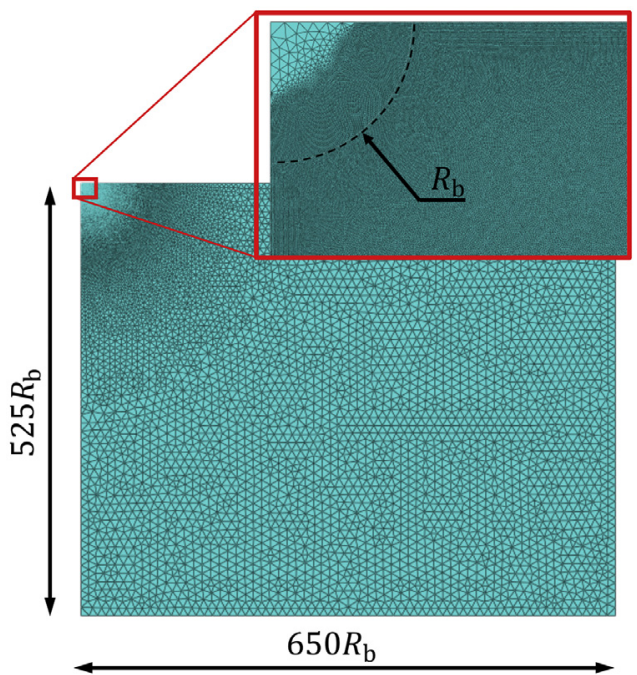

Fig. 3. FEM mesh used for the simulation. A gradient strategy is used to accurately capture the stress distribution during contact process. A sufficiently large computation domain is modeled to eliminate the free boundary effect.

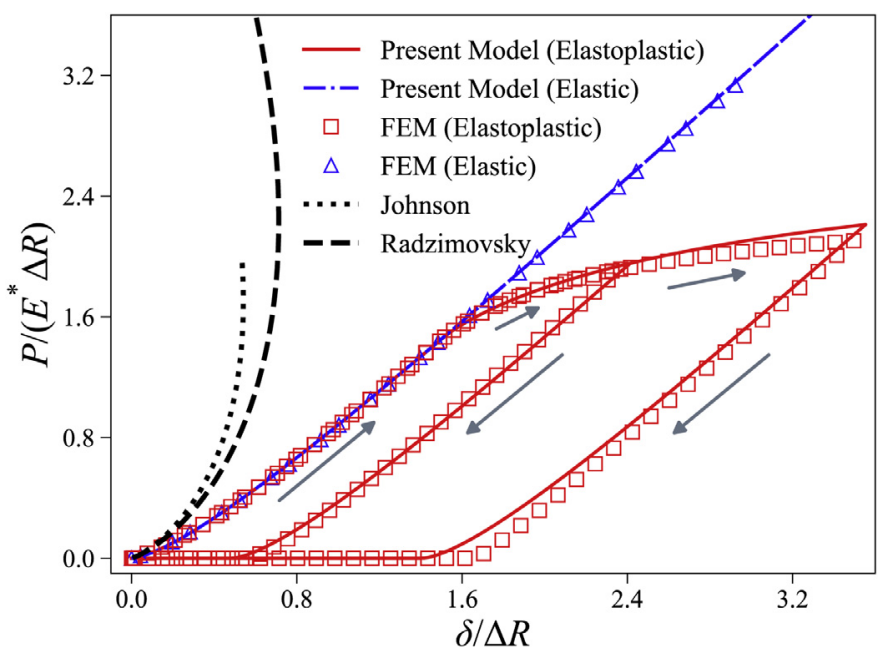

Fig. 4. Elastoplastic $(\mathrm{E}+\mathrm{P})$ model used for conformal contact. The coefficient $k$ was set as 0.663 to fit the model with the FEM data.

distribution, with a finest element size of $1 \% R_{\mathrm{b}}$. The mesh sensitivity was checked, and the results indicated that the present mesh was sufficiently accurate.

According to Liu et al. [28], and our previous study [41], during the contact process, the contact pressure distribution has a specific form depending on the contact geometry, i.e., sphere-to-sphere or cylinder-to-cylinder. Also, the peak value of that distribution can be assumed to be linear with respect to the compressive displacement, where an undetermined coefficient $k$ emerges as a factor that can be further determined by fitting Eq. (1) according to the FEM results. In the present case, $k$ is set to 0.663 to fit the present model with the FEM data. As shown in Fig. 4, the NFD relationships of the models of Johnson and Radzimovsky exhibit a parabolic relationship between the load and displacement; this relationship may lead to unphysical characteristics when the loading displacement or load is large. It is also evident that when the compressive displacement is larger than the critical value, these two models deviate from the FEM results. On the contrary, our model agrees with the FEM results in both elastic and plastic cases.

\subsection{Dynamics with plasticity, damping, and friction}

Any initial state $\left(\boldsymbol{r}_{0}, \boldsymbol{V}_{0}\right)$ of the journal located at location $O_{0}$ (shown in Fig. $5 \mathrm{a}$ ) uniquely determines the state before collision. The journal moves freely to point $O_{1}$, where the journal impacts the bearing at the contact point $C_{1}$ with an approaching angle $\alpha_{\mathrm{a} 1}$ (Fig. 5b). After the journal departs from the bearing with a departure angle $\alpha_{\mathrm{d} 1}$, it moves from location $O_{1}$ to location $O_{2}$, where the second impact takes place at point $C_{2}$ (Fig. $5 \mathrm{c}$ ). We denote the location of the journal 


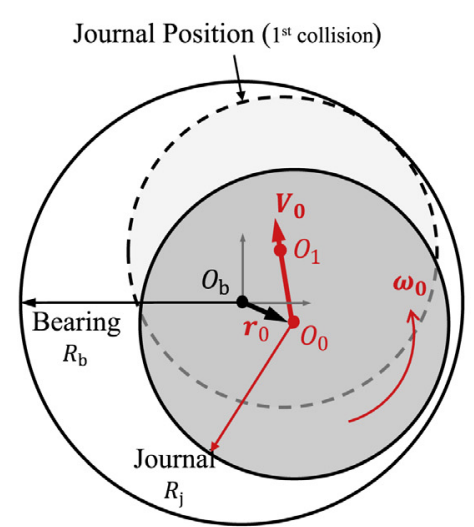

a)

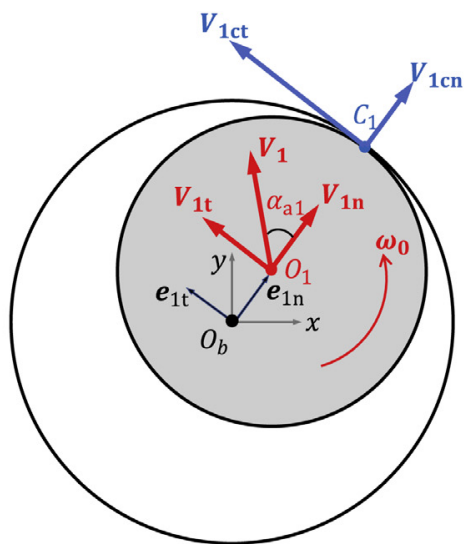

Journal Position (2nd collision)

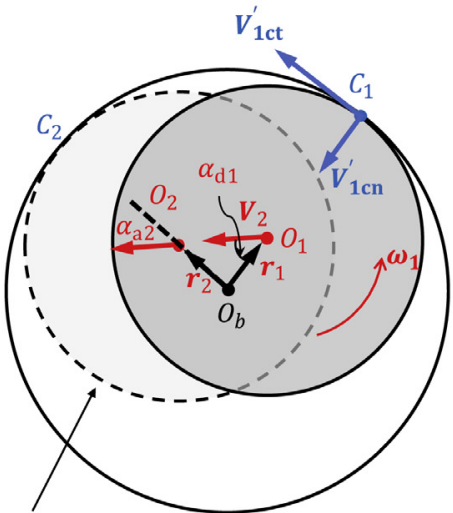

c)

Fig. 5. Schematic diagrams of the first two collisions. a) Initial state of the journal, b) state before collision, and c) state after collision.

at the $i$ th impact with $O_{i}$, and the impact point with $C_{i}$. Geometrically, we have $\left|O_{b} C_{i}\right|=\left|O_{b} C_{i+1}\right|$, and hence $\alpha_{\mathrm{d} i}=\alpha_{\mathrm{a}(i+1)}$. During the impact process, we assume that the time for the whole process can be neglected. Consequently, we can formulate and solve the impact process and inertial motion separately and independently.

First, the free motion between two consecutive collisions is modeled. From the starting point $O_{i}$ to the ending point $O_{i+1}$, the angular velocity $\omega_{i}$ and velocity $\boldsymbol{V}_{i}$ remain constant in the absence of an external force. The ending position vector can easily be obtained by associating the motion equation and geometrical condition for contact as follows:

$$
\left\{\begin{array}{l}
\boldsymbol{r}_{i+1}=\boldsymbol{r}_{i}+\boldsymbol{V}_{i} t_{\mathrm{f}} \\
\left\|\boldsymbol{r}_{i+1}\right\|=\Delta R
\end{array}, \text { for } t_{\mathrm{f}}>0\right.
$$

where $\boldsymbol{r}_{\boldsymbol{i}}$ is the position vector of the journal when the $i$ th collision takes place, $t_{\mathrm{f}}$ is the time of free motion for two consecutive collisions, and $\Delta R$ is the difference $R_{\mathrm{b}}-R_{\mathrm{j}}$. Since the starting point always resides in the inner domain of circle $R_{\mathrm{b}}$, the uniqueness of Eq. (6) is ensured.

Second, the impacting process is modeled. At the time of collision $i$, the impacting velocity $\boldsymbol{V}_{i}$ can be decomposed into a normal direction of impact $\boldsymbol{V}_{i n}$ and a tangent direction $\boldsymbol{V}_{i t}$ We denote the unit vector along these two velocity vectors as $\boldsymbol{e}_{i n}$ and $\boldsymbol{e}_{i t}$, respectively (Fig. 5b). We obtain $\boldsymbol{e}_{i n} \| \boldsymbol{r}_{\boldsymbol{i}}$ and $\boldsymbol{e}_{i \mathrm{t}} \perp \boldsymbol{r}_{i}$. The impacting force $P_{i}$ as a function of the compressive displacement $\delta_{i}$ consists of two parts, one of which is related to body deformation and the other to velocity-related damping. The expression for the dynamic NFD relationship can be rewritten as follows:

$$
P= \begin{cases}\left(k E^{*}\left(\frac{2}{3} \delta^{\frac{3}{2}}-g^{\frac{1}{2}} \delta+\frac{1}{3} g^{\frac{3}{2}}\right)+R \sigma_{Y} g^{\frac{1}{2}}\right) \sqrt{\frac{8}{\Delta R+\delta}}+D \dot{\delta}, & \text { if } g \geq 0 \text { and } \dot{\delta}>0 \\ \frac{8}{3} k E^{*} \delta \sqrt{\frac{\delta}{2(\Delta R+\delta)}+D \dot{\delta},} & \text { if } g<0 \text { and } \dot{\delta}>0 \\ \frac{8}{3} k E^{*}\left(\delta-\delta_{p}\right) \sqrt{\frac{\delta-\delta_{p}}{2\left(\Delta R+\delta-\delta_{p}\right)}}+D \dot{\delta}, & \text { if } \dot{\delta} \leq 0\end{cases}
$$

where the condition $g \geq 0$ and $\dot{\delta}>0$ corresponds to plastic loading, the $\dot{\delta} \leq 0$ corresponds to unloading, $\delta_{p}$ is the plastic deformation, and

$$
D=\mu \delta^{\frac{3}{2}}
$$

also known as the damping coefficient. To complete this formulation, the damping coefficient $D$ or hysteresis damping factor $\mu$ must be determined. A good approach was provided by Lankarani and Nikravesh[22]. The hysteresis damping factor $\mu$ can be related to the stiffness of the indentation $K_{0}$ according to the following equation:

$$
\mu=\frac{3 K_{0}\left(1-c_{\mathrm{e}}^{2}\right)}{4 V_{0}^{\perp}}
$$

where $V_{0}^{\perp}$ is the normal component of the impact velocity, $V_{0}$, and $c_{\mathrm{e}}$ is the estimated restitution coefficient. The stiffness of the indentation $K_{0}$ can be obtained at small values of compressive displacement, as follows:

$$
K_{0}=\frac{8}{3} k E^{*} \sqrt{\frac{1}{\Delta R}}
$$

Eq. (7) governs the dynamic contact process. Because the nonlinear property includes plasticity and damping, it is difficult to obtain an analytical closed-form solution for $\delta(t)$ and $\dot{\delta}(t)$. Consequently, the numerical integration method RK45 [45,46] 
was used to solve the dynamic process. By numerically integrating Eq. (7), we can obtain the historical results for $P_{i}(t)$, $\delta_{i}(t)$ and $\dot{\delta}_{i}(t)$. Finally, we have the normal departure velocity $\boldsymbol{V}_{i c n}^{\prime}=\dot{\delta}_{i} \boldsymbol{e}_{i n}$ at the detaching time. Then, we model the sliding behavior during impact, for which a friction model must be introduced. Pennestri et al. [47] reviewed eight friction models commonly used in the study of dynamics. A more complex model, such as the friction model of Gonthier [48], can produce more realistic phenomena such as stiction, static force, and pre-sliding. However, for simplicity, the Coulomb friction model was an approach of choice in recent studies, such as those of Issac et al. [49] and Qian et al. [50]. In the present study, in which plasticity is main focus, we used the Coulomb friction model. As the bearing stays fixed, the Coulomb friction $\boldsymbol{f}_{i}$ can be determined by the normal force and the relative speed $\boldsymbol{V}_{i c t}=\boldsymbol{V}_{i \mathrm{t}}+R_{j} \boldsymbol{\omega}_{i} \times \boldsymbol{e}_{i \mathrm{n}}$, which can be formulated as follows:

$$
\boldsymbol{f}_{i}=-\left(\operatorname{sign}\left(\boldsymbol{V}_{i c t} \cdot \boldsymbol{e}_{i \mathrm{t}}\right)\right) \mu_{f}\left|P_{i}\right| \boldsymbol{e}_{i \mathrm{t}}
$$

where $\mu_{f}$ is the coefficient of friction and $\operatorname{sign}(\cdot)$ is the signum function that maps negatives to -1 , zero to 0 , and positives to 1 . The departing velocity and angular velocity can be then calculated by the following equations.

$$
\boldsymbol{V}_{i+1}=\boldsymbol{V}_{i t}+\frac{1}{m} \int_{0}^{t} \boldsymbol{f}_{i} \mathrm{~d} t+\dot{\delta}_{i} \boldsymbol{e}_{i n}
$$

and

$$
\boldsymbol{\omega}_{i+1}=\boldsymbol{\omega}_{i}+\frac{1}{J} \int_{0}^{t} R_{j} \boldsymbol{e}_{i n} \times \boldsymbol{f}_{\mathrm{i}} \mathrm{d} t
$$

\section{Results and discussion}

The parameters for the presented study are as follows: the constant $k$ is 0.663 , the equivalent elastic modulus $E^{*}$ is $100 \mathrm{GPa}$, the yield stress $\sigma_{\mathrm{Y}}$ is $100 \mathrm{MPa}$, the radius of the bearing $R_{\mathrm{b}}$ is $0.1 \mathrm{~m}$, the radial difference $\Delta R$ is $0.001 R_{\mathrm{b}}$, the mass of the journal $m$ is $200 \mathrm{~kg}$, and the moment of inertia $J$ is $1 \mathrm{~kg} \bullet \mathrm{m}^{2}$. Simulations using different models, i.e., elasticdamping model $(E+D)$, elastoplastic model $(E+P)$, and elasto-plasto-damping model $(E+P+D)$, were carried out to study the effect of plasticity. In particular, one-dimensional (1D) simulations were used to study the evolution of impact velocity under consecutive collisions and two-dimensional (2D) simulations were performed to investigate the effects of plasticity on journal motion.

\subsection{Critical speed}

The impact speed can be referred to as the critical speed at which the journal reaches the plastic zone, ignoring any velocity-related damping effects. The critical speed $V_{\mathrm{cr}}$ can be obtained by the following equations.

$$
\frac{1}{2} m V_{\mathrm{cr}}^{2}=\int_{0}^{\delta_{\mathrm{cr}}} \frac{8}{3} k E^{*} \delta \sqrt{\frac{\delta}{2(\Delta R+\delta)}} \mathrm{d} \delta
$$

and

$$
\bar{V}=\frac{V}{V_{\text {cr }}}
$$

Assuming $\delta \geq 0$ and $\Delta R>0$, by integrating Eq. (14), the explicit expression of $V_{\text {cr }}$ can be found as follows:

$$
V_{\mathrm{cr}}=\left(\frac{2 \sqrt{2}}{3 m} k E^{*}\left(\sqrt{\frac{\delta_{\mathrm{cr}}}{\Delta R+\delta_{\mathrm{cr}}}}\left(2 \delta_{\mathrm{cr}}^{2}-\Delta R \delta_{\mathrm{cr}}-3 \Delta R^{2}\right)+3 \Delta R^{2} \ln \left(\frac{\sqrt{\Delta R+\delta_{\mathrm{cr}}}+\sqrt{\delta_{\mathrm{cr}}}}{\sqrt{\Delta R}}\right)\right)\right)^{\frac{1}{2}}
$$

On the other hand, due to friction, the journal will spin as it collides with the bearing; the angular speed can be nondimensionalized with the kinetic energy at the initial state, as follows:

$$
\bar{\omega}=\frac{\omega}{\omega_{\text {ref }}}
$$

where

$$
\omega_{\text {ref }}=\sqrt{\frac{m}{J}} V_{0}
$$

Using Eq. 16, we can obtain the critical speed for regular heavy-load bearings. Consider SKF bearings, for example. Their dimensions (including clearances) are typically designed in accordance with the ISO 12240-1, or ANSI/ABMA standards, which provide the dynamic loading limits. Using these values, the critical velocity can be calculated and plotted as a function of equivalent dynamic payload (Fig. 6). Such a graph can guide the design of a revolute joint for heavy loading cases when plasticity is an important factor that must be considered. Note that, for most of the SKF bearings under the maximum dynamic payload, plasticity should be taken into account if the contact velocity exceeds $1.5 \mathrm{~m} / \mathrm{s}$. 


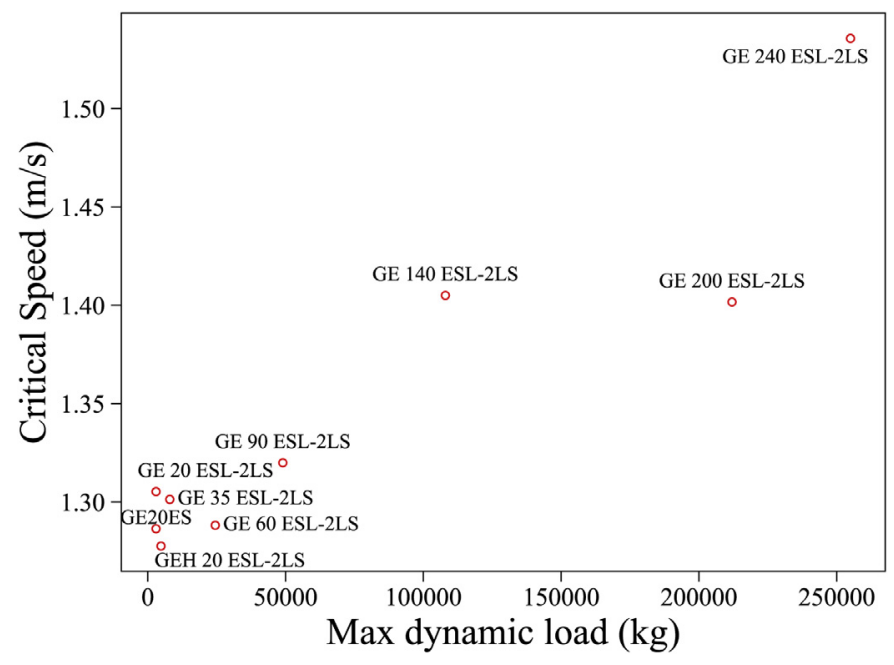

Fig. 6. Design chart indicating the critical speeds for several SKF bearings.

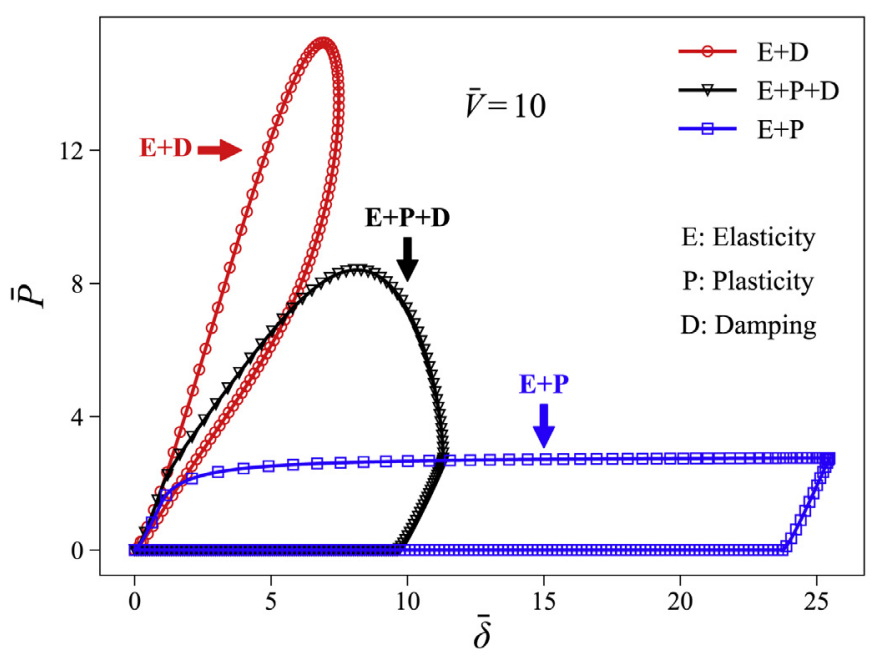

Fig. 7. Force-compressive displacement relationship for various models.

\subsection{Impact process}

The collision between the journal and the bearing plays an important role in the dynamical study. In this section, we first investigate the impact process during a single collision. Fig. 7 shows the contact force as a function of the compressive displacement under an impact velocity $\bar{V}_{0}=10$. For the $\mathrm{E}+\mathrm{D}$ model, the only dissipation is related to damping, which is about $44.0 \%$ of the initial kinetic energy. On the contrary, for the models involving plasticity, the energy dissipation is significantly boosted; in the $\mathrm{E}+\mathrm{P}$ model, about $96.6 \%$ of the initial kinetic energy is dissipated by plastic deformation, while in the $\mathrm{E}+\mathrm{P}+\mathrm{D}$ model, the dissipation is about $96.9 \%$. For the $\mathrm{E}+\mathrm{P}$ model, since the damping part of the restoring force vanishes, the physics of such a model should be equivalent to a quasi-static contact process, which was discussed in Section 2.1 .

Energy dissipation results in inelastic collision. Fig. 8 shows the relationship between the restitution coefficient $c_{\mathrm{e}}$ and the dimensionless impact speed $\bar{V}_{0}$. The red line represents the E+D model. The estimated restitution coefficient (i.e., the input $c_{\mathrm{e}}$ ) is set at 0.9 to calculate the hysteresis damping factor $\mu$. The simulated restitution coefficient (i.e., the output $c_{\mathrm{e}}$ ) is close to 0.9 at low impact speed, which is in agreement with the input-output relationship reported by Lankarani and Nikravesh [22]. The blue line represents the E+P model without taking velocity-related damping into account. If $\bar{V}_{0} \leq 1$, the journal will bounce back without loss of any energy, which indicates a pure elastic range. If $\bar{V}_{0}>1$, plastic deformation will take place. As the impact speed increases, the restitution coefficient decreases drastically because of plastic dissipation. For the $\mathrm{E}+\mathrm{P}+\mathrm{D}$ model, the critical impact velocity that distinguishes elastic and plastic deformation will be slightly higher than $V_{\mathrm{cr}}$, since the damping force will dissipate a fraction of the kinetic energy. The inset of Fig. 8 shows a delayed separation 


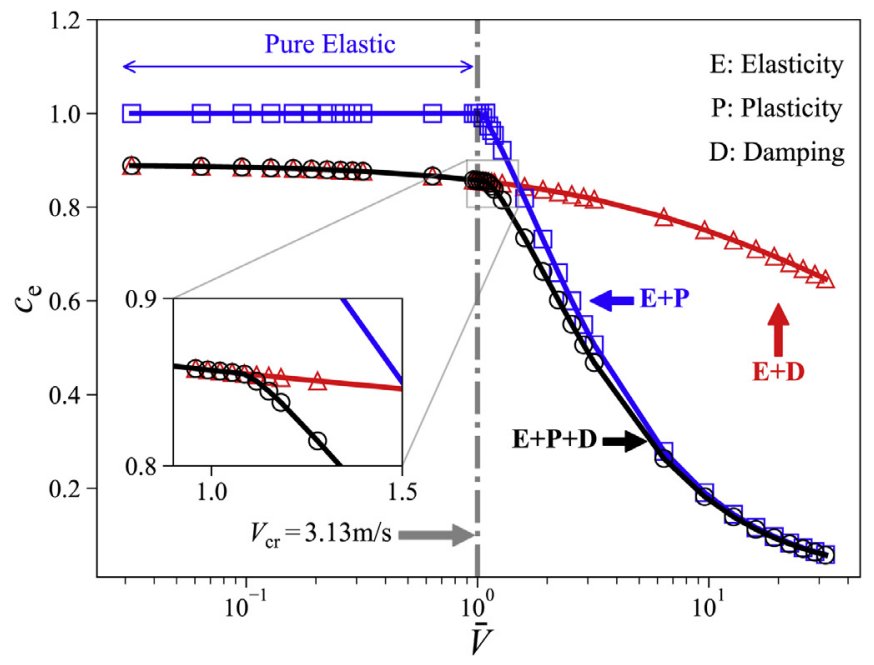

Fig. 8. Restitution coefficient as a function of dimensionless impact velocity. The inset is a magnification of the area near $\overline{\mathbf{V}}=1$ for the $E+P+D$ and $E+D$ models. A velocity slightly larger than $\boldsymbol{V}_{\mathbf{c r}}$ is evident.

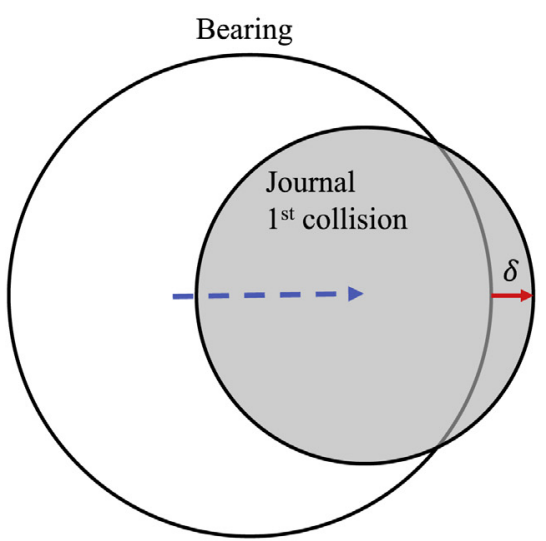

a)

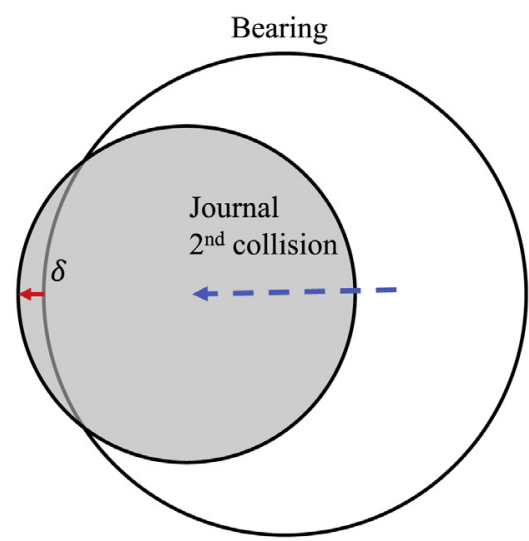

b)

Fig. 9. One-dimensional consecutive collisions. a) The first collision on one side of the bearing and b) the second collision on the other side.

between the $\mathrm{E}+\mathrm{D}$ model and $\mathrm{E}+\mathrm{P}+\mathrm{D}$ models at a speed slightly greater than $V_{\mathrm{cr}}$, which proves the assertion. Similarly, for low impact velocity, plastic deformation will not take place, so the journal motion is exactly the same as that simulated with the E+D model. However, at high impact speed, the energy dissipation by plastic deformation is the main reason why $c_{\mathrm{e}}$ drops quickly. Additionally, because of the damping effect, extra energy dissipation is added to the total energy loss in comparison with the E+P model.

\subsection{One-dimensional cases}

Trivial 1D cases were used to study the consecutive impact behavior of the journal (Fig. 9). In these cases, both the approaching angle and the initial angular velocity of the journal vanish. The journal is moving back and forth between two symmetrical contact points. On the other hand, from a geometrical point of view, for 2D planar motion of the journal, the magnitude of the $(i+1)$ th normal impact velocity is always identical to the ith normal departure velocity, namely, $V_{(i+1) \mathrm{n}}=V_{i \mathrm{n}}^{\prime}$, since the approaching angle of the $(i+1)$ th collision and the departure angle of the $i$ th collision are identical. The velocity variation in this $1 \mathrm{D}$ case can be used to predict the normal velocity variation in a $2 \mathrm{D}$ case.

Fig. 10a shows phase portraits with initial velocity $\bar{V}_{0}=10$ for the E+D and E+P+D models. The abscissa is related to the compressive displacement instead of the actual location, to eliminate free motion of the journal between every two consecutive collisions. In the $\mathrm{E}+\mathrm{D}$ model, the state of the journal approaches a spiral sink. In the $\mathrm{E}+\mathrm{P}+\mathrm{D}$ model, the first collision leads to significant plastic deformation. Because of material yielding, a lower-magnitude restoring force is exerted on the journal than in the E+D model. Consequently, the largest compressive displacement for the first collision is considerably 

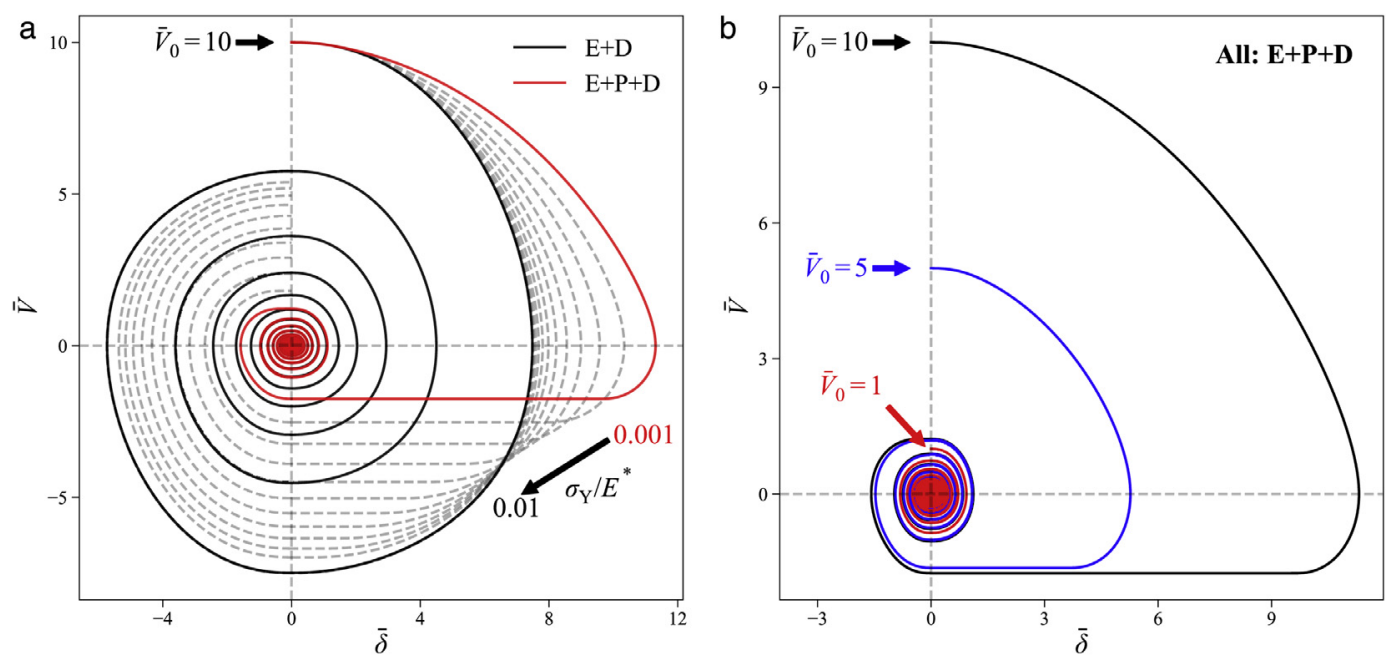

Fig. 10. Phase portraits. a) Comparison of the elasto-damping $(E+D)$ and the elasto-plasto-damping $(E+P+D)$ models. The grey dashes represent continuous deformation from $\mathrm{E}+\mathrm{P}+\mathrm{D}\left(\boldsymbol{\sigma}_{\mathbf{Y}} / \boldsymbol{E}^{*}=0.001\right)$ model to $\mathrm{E}+\mathrm{P}$ model. b) Elasto-plasto-damping $(\mathrm{E}+\mathrm{P}+\mathrm{D})$ model for various initial states.

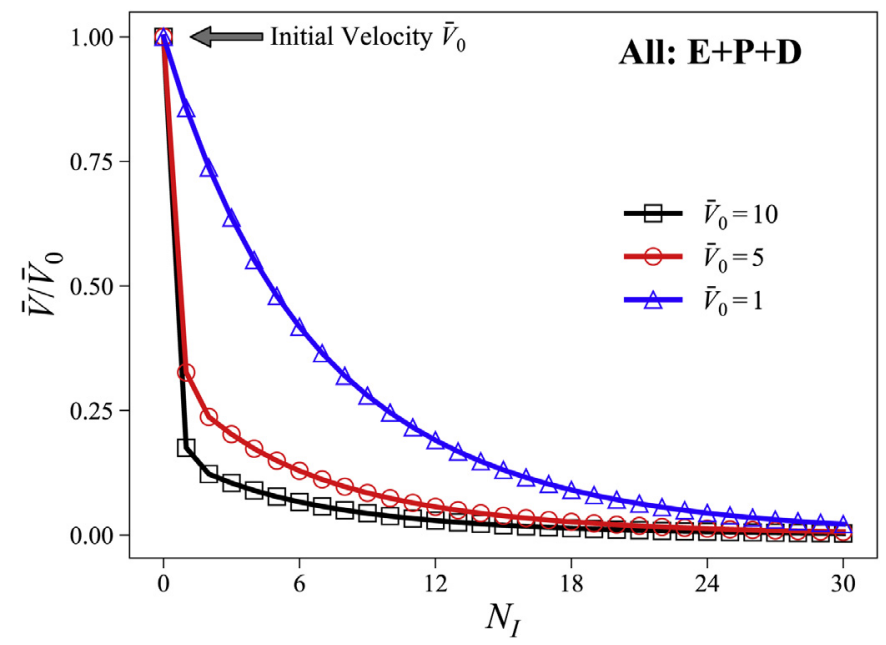

Fig. 11. Remaining velocity as a function of consecutive collisions according to the elasto-plasto-damping (E+P+D) model.

larger than in the $\mathrm{E}+\mathrm{D}$ model. Besides, since more energy is dissipated by plastic work, the state of the journal falls much more rapidly toward the spiral sink, until the $\mathrm{E}+\mathrm{P}+\mathrm{D}$ model turns into an equivalent of the $\mathrm{E}+\mathrm{D}$ model. In fact, as shown by the grey dashes (with $\sigma_{\mathrm{Y}} / E^{*}$ chosen as $0.0015,0.0020,0.0025, \ldots, 0.01$ ) in Fig. 10a, if we continuously increase the value of $\sigma_{\mathrm{Y}} / E^{*}$, the phase trajectory will continuously deform from that of the $\mathrm{E}+\mathrm{P}+\mathrm{D}$ model with $\sigma_{\mathrm{Y}} / E^{*}=0.001$ to that of the $\mathrm{E}+\mathrm{D}$ model, where the yielding stress is sufficiently large such that plastic deformation never happens. Mathematically, the continuous deformation between the red and the black trajectories indicates that there is a homotopy between them. The embedding parameter $\sigma_{\mathrm{Y}} / E^{*}$ can be taken as the homotopy parameter in this family of phase trajectories. Fig. 10b presents the phase states for the $\mathrm{E}+\mathrm{P}+\mathrm{D}$ model for various initial impact velocities. For an impact speed higher than the critical speed, plastic dissipation plays a major role in the first few collisions, until the impact velocity falls into the range of the elastic zone. The ratio of $\bar{V}$ to $\bar{V}_{0}$ as a function of the number of consecutive collisions is plotted Fig. 11 . For $\bar{V}_{0} \leq 1$, the impact velocity decreases at a ratio of about 0.9 . For $\bar{V}_{0}>1$, the impact velocity drops quickly until it reaches the E+D zone (Fig. 11b).

\subsection{Two-dimensional cases}

In this section, we examine the effect of initial conditions on dynamic impact in the single journal-bearing system in 2D. Simulations with different initial angles were performed for the $\mathrm{E}+\mathrm{D}$ and $\mathrm{E}+\mathrm{P}+\mathrm{D}$ models. Because of the axial symmetry 

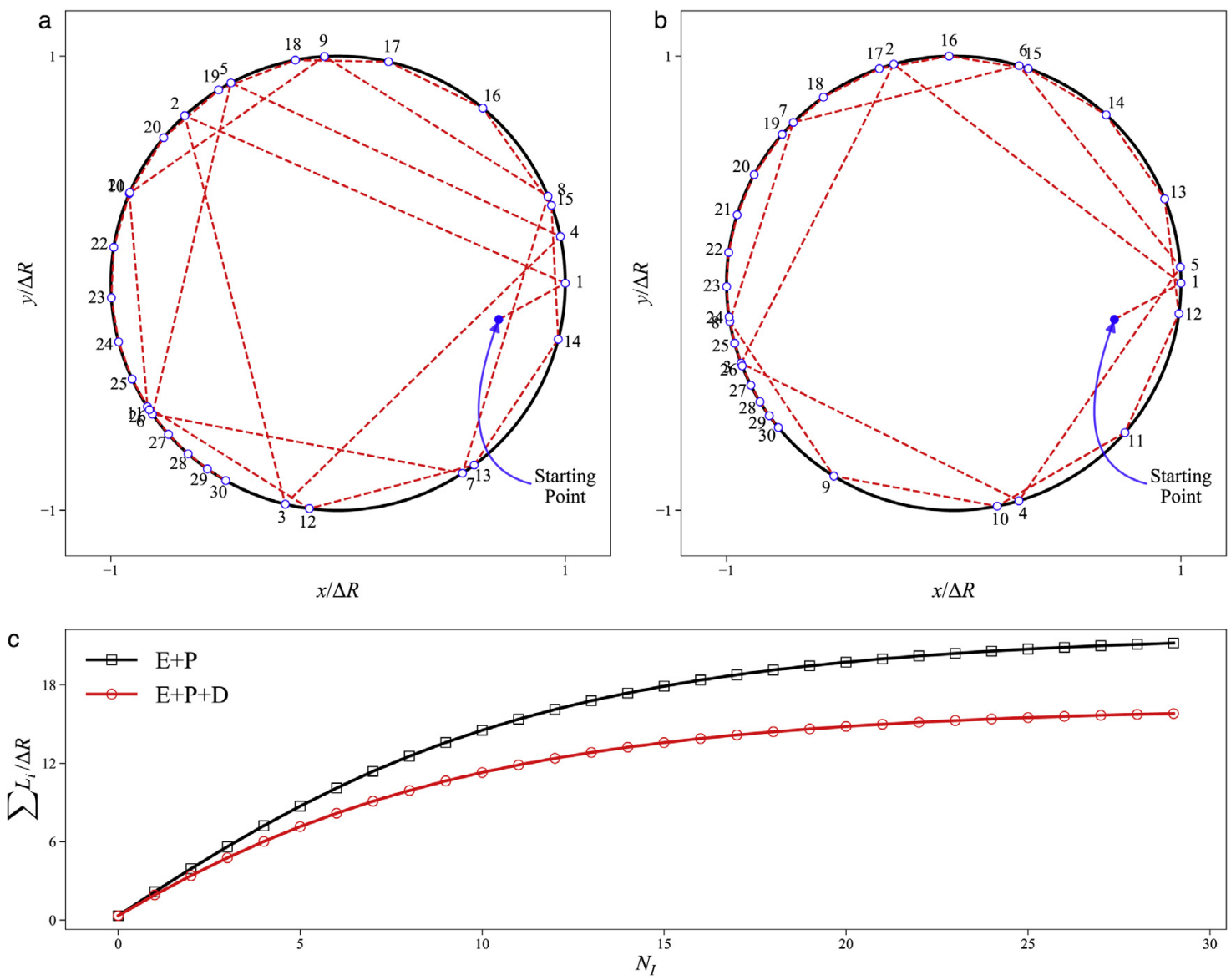

Fig. 12. Simulated trajectories. a) Elasto-damping $(E+D)$ model and b) elato-plasto-damping $(E+P+D)$ model. The journal starts with its center located at the starting point. The collision points are numbered sequentially.

of the bearing, without loss of generality, we can fix the first contact point on the $x$-axis, as indicated by touch point 1 in Fig. 12.

Fig. 12a and $\mathrm{b}$ illustrate the trajectories of the center of the journal simulated with the $\mathrm{E}+\mathrm{D}$ and $\mathrm{E}+\mathrm{P}+\mathrm{D}$ model. The dimensionless impact velocity is $\bar{V}_{0}=3.2$ and the approaching angle is $\alpha_{\mathrm{a} 0}=0.498$ rad. First, the journal moves from the starting point to collision point 1 . The trajectories from collision point 1 to point 30 are distinctly different because of differences in energy loss between the two models. The secant between two consecutive collision points in the E+P+D model is generally shorter than that in the $\mathrm{E}+\mathrm{D}$ model. Denoting $L_{i}$ as the length of the secant from point $i$ to point $i+1$, the total travel distance of the journal can be obtained by summing over all collisions. Fig. 12c compares the total travel distance among the various models. The absence of plasticity in the $\mathrm{E}+\mathrm{D}$ model increases the total travel distance by about $34 \%$.

The reason for such difference is that the change in normal velocity differs between the E+D and E+P+D models, which results in variation in the departure angel after collisions. To understand such trajectorial differences, the following parameters were investigated: energy variations, angular velocity, tangent velocity, and the approach and departure angle. Since the normal departure velocity of any collision is identical to the approaching velocity of the next collision, the evolution of the normal velocity is equivalent to the 1D case, as discussed in Section 3.3.

The energy variations are visualized by the stacking bar plots in Fig. 13. The energies above the abscissa are transitional and rotational kinetic energies. The energies below the abscissa are in the form of energy dissipation caused by friction and deformation/damping. As the journal repeatedly collides with the bearing, the transitional energy is simultaneously dissipated by friction, plastic deformation, and damping. First, the normal component of the impact velocity is damped by deformation. If plasticity is involved, that part of the energy is dissipated much faster. As noted in Section 3.3, the tangent movement is dragged by friction; meanwhile, the journal begins to rotate so the rotatory part of the kinetic energy increases. Although plastic deformation generally contributes more to the total dissipation than does velocity-related damping, the actual normal restoring force exerted on the journal is smaller than that in the E+D model (Fig. 7). As a result, the friction during the first collision in the $\mathrm{E}+\mathrm{D}$ model is greater than that in the $\mathrm{E}+\mathrm{P}+\mathrm{D}$ model. This explains why the dissipation by 

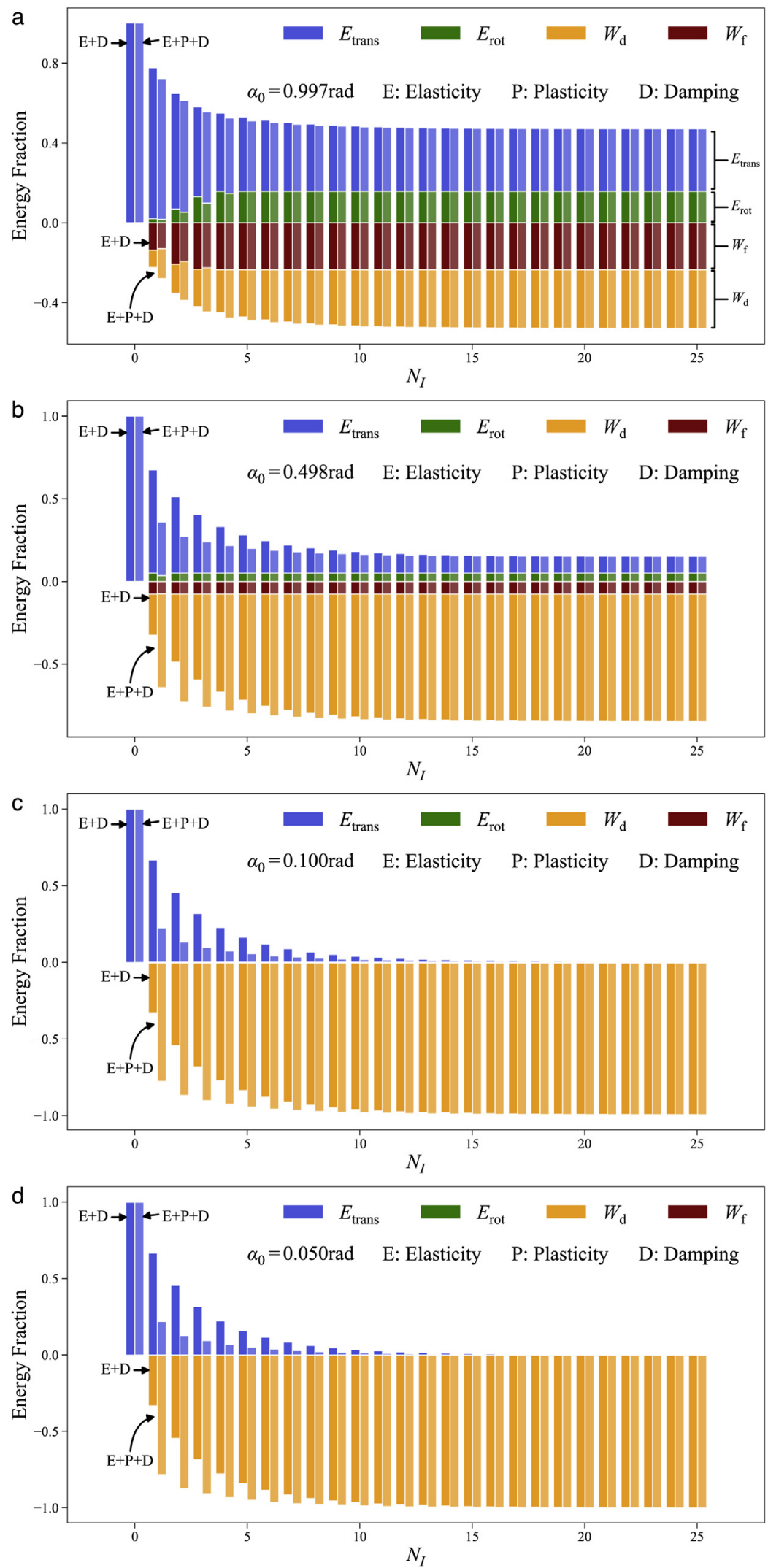

Fig. 13. Energy variations as a function of consecutive collisions for the elasto-damping $(E+D)$ and elasto-plasto-damping $(E+P+D)$ models at different initial impact angles. 

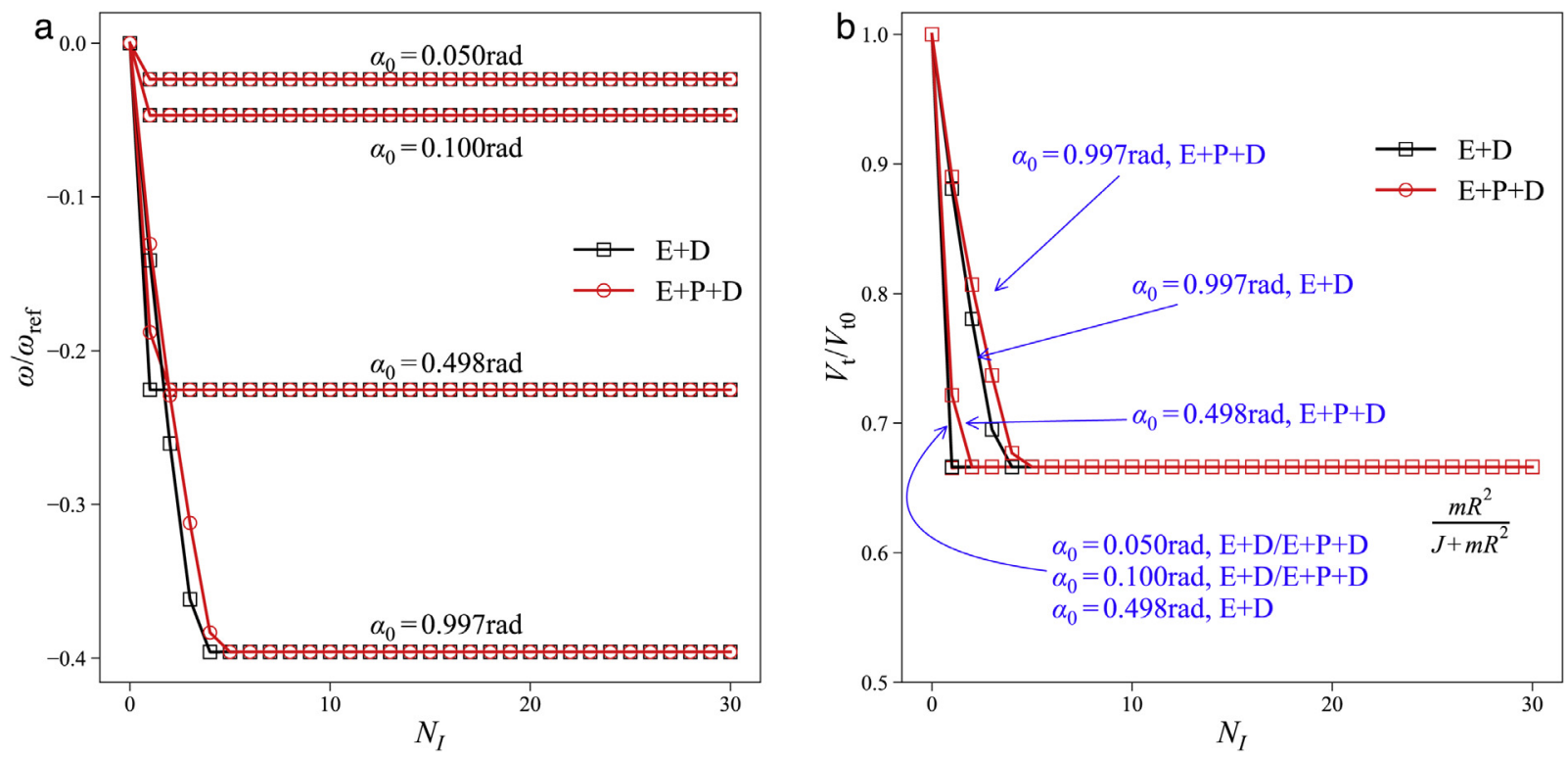

Fig. 14. a) Journal angular velocity and b) tangent velocity as a function of the number of collisions.

friction in the $\mathrm{E}+\mathrm{D}$ model tends to stabilize more quickly than in the $\mathrm{E}+\mathrm{P}+\mathrm{D}$ model. The variation in the rotatory part of kinetic energy behaves similarly.

Summarizing, comparing the E+P+D and E+D models, we found that plastic and damping dissipation stabilize more quickly in the $\mathrm{E}+\mathrm{P}+\mathrm{D}$ model, whereas frictional dissipation stabilizes more quickly in the $\mathrm{E}+\mathrm{D}$ model. On the other hand, when the initial approaching angle is greater, the frictional effects is much more significant. At a small approaching angle, dissipation caused by friction and rotatory kinetic energy represents a very small fraction of the total energy.

Due to friction, the tangent component of the velocity continues to decline and the journal continues to spin up. The angular velocity and tangent velocity can be obtained as follows:

$$
\omega=\frac{1}{J} \int_{0}^{t} f R \mathrm{~d} t
$$

and

$$
V_{\mathrm{t}}=V_{\mathrm{t} 0}+\frac{1}{m} \int_{0}^{t} f \mathrm{~d} t
$$

where $V_{\mathrm{t} 0}$ is the tangent velocity at $t=0$. While friction is a function of the relative speed at the contacting surface, friction vanishes when it meets the following condition:

$$
V_{\mathrm{t}}+\omega R=0
$$

Substituting Eqs. (18) and (19) into Eq. (20), one can obtain the following relationship for the invariant terminal tangent velocity and angular velocity where friction vanishes:

$$
\begin{aligned}
& \frac{V_{\mathrm{t}}}{V_{\mathrm{t} 0}}=\frac{m R^{2}}{J+m R^{2}} \\
& \omega=-\frac{m R}{J+m R^{2}} V_{\mathrm{t} 0}
\end{aligned}
$$

The ratio of the terminal tangent velocity to its initial value is constant if the values of $m, R$, and $J$ are given. It is independent of the initial value and choice of model (E+P+D or E+D). Fig. 14 plots the variation of the angular and tangent velocity as a function of the number of collisions for various initial approaching angles. In both models, the value of $V_{\mathrm{t}} / V_{\mathrm{t} 0}$ reaches to the same terminal value after a few collisions. Since $\omega$ depends on $V_{\mathrm{t} 0}$, for a larger impact angle, the terminal value of $\omega$ is generally larger (Fig. 14a). On the other hand, for a small approaching angle, so that the tangent component of the velocity is smaller while the friction is larger, the stable state is achieved in a very short time. For example, when the initial approach angle is small, the stable state is achieved during the first collision, while in some larger-angle cases, this process requires 3-5 collisions.

The secant length illustrated in Fig. 12 is directly related to departure angle by $L_{i}=2 \Delta R \cos \alpha_{\mathrm{d} i}$. Therefore, the variation of departure angle is investigated to understand the variation of secant length during successive collisions. The departure angle 


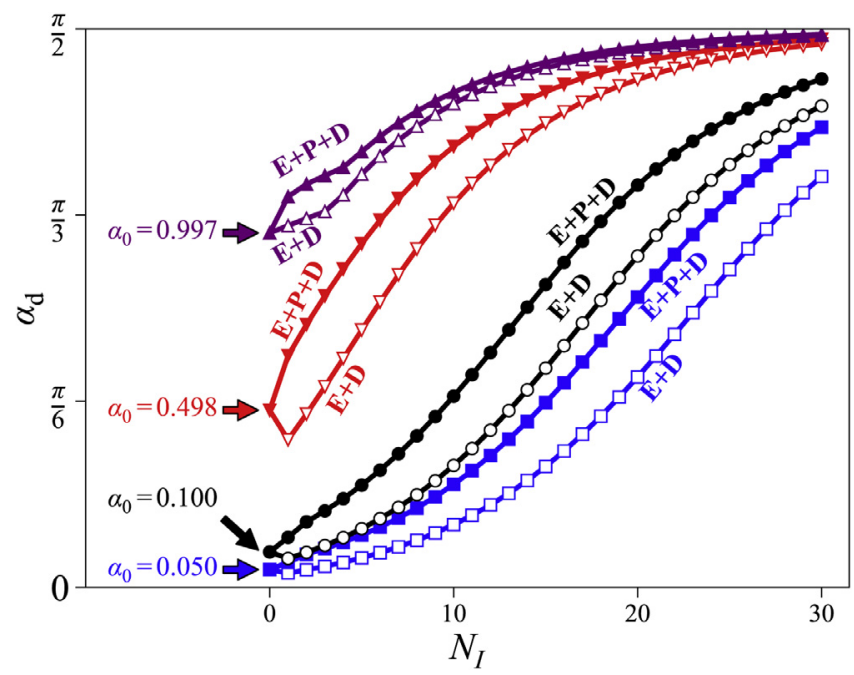

Fig. 15. Departure angle as a function of the number of collisions in the different models.

of the $i$ th collision can be expressed as $\alpha_{\mathrm{d}}=\arctan \left(\frac{V_{\mathrm{ti}}}{V_{\mathrm{n}}}\right)$. As proved before, the term $V_{\mathrm{t} i}$ will be reduced to a nonzero constant if $\alpha_{\mathrm{a} 0}$ is nonzero, while the term $V_{\mathrm{n} i}$ will be damped by every collision and asymptotes to 0 . As a result, the departure angle will asymptote to $\pi / 2$, as shown in Fig. 15. Although the long-term trends are similar for the $E+D$ and $E+P+D$ models, there are differences at the first few collisions. In the $\mathrm{E}+\mathrm{P}+\mathrm{D}$ model, the departure angle increases monotonically, while in the $\mathrm{E}+\mathrm{D}$ model, it decreases initially and then increases, especially for the case with a small initial approach angle. The decreasing of the departure angle suggests that the ratio of the loss of the normal speed is smaller than that of the tangent speed. In the $\mathrm{E}+\mathrm{D}$ model, the ratio of the loss of normal velocity is about 0.2. If the tangent velocity achieves its terminal value during the first collision, the ratio of the loss of the tangent velocity is $\frac{J}{J+m R_{\mathrm{b}}^{2}} \approx 0.66$. In that case, the departure angle will be smaller than the approaching angle. Next, we consider a condition where the departure angle is smaller than the approaching angle during a single collision. Generally, the normal and tangent velocity can be expressed as follows:

$$
V_{\mathrm{n} 1}=-V_{\mathrm{n} 0}+\frac{1}{m} \int_{0}^{t_{\mathrm{c}}} P \mathrm{~d} t
$$

where $t_{\mathrm{c}}$ is the contact time and the minus sign indicates that the journal moves away from the bearing center, and

$$
V_{\mathrm{t} 1}=V_{\mathrm{t} 0}-\frac{1}{m} \int_{0}^{t_{f}} \mu_{f} P \mathrm{~d} t
$$

where $t_{f}$ is the time when the friction vanishes and $t_{f} \leq t_{\mathrm{c}}$. Comparing Eqs. (23) and (24), the following inequality can be obtained:

$$
\frac{V_{\mathrm{t} 0}-V_{\mathrm{t} 1}}{\mu_{f}} \leq V_{\mathrm{n} 1}+V_{\mathrm{n} 0}
$$

Then, using $\tan \alpha_{d}=V_{\mathrm{t} 1} / V_{\mathrm{n} 1}$ and $\tan \alpha_{\mathrm{a}}=V_{\mathrm{t} 0} / V_{\mathrm{n} 0}$, the following relationship can be obtained:

$$
\tan \alpha_{\mathrm{d}}-\tan \alpha_{\mathrm{a}} \geq \frac{1}{c_{\mathrm{e}}}\left(\left(1-c_{\mathrm{e}}\right) \tan \alpha_{\mathrm{a}}-\mu_{f}\left(1+c_{\mathrm{e}}\right)\right)
$$

As a result, $\tan \alpha_{\mathrm{d}} \leq \tan \alpha_{\mathrm{a}}$ implies the following inequality.

$$
\tan \alpha_{\mathrm{a}} \leq \mu_{f} \frac{1+c_{\mathrm{e}}}{1-c_{\mathrm{e}}}
$$

Notably, in the presented cases, $\tan \alpha_{\mathrm{a}} \approx 9 \mu_{f}$ is the critical value for the $\mathrm{E}+\mathrm{D}$ model.

The journal behavior is strongly dependent on the initial state, which is characterized by the magnitude of the velocity and the approaching angle of the first collision. State variations for different initial conditions and models are plotted in Fig. 16. The total domain can be divided into two different parts, one of which is the plastic zone, wherein plastic deformation takes place such that the $\mathrm{E}+\mathrm{P}+\mathrm{D}$ model should be more accurate. The gray square markers represent the change in status simulated by the $\mathrm{E}+\mathrm{D}$ model; the critical condition for the states going left first and then right is $\tan \alpha_{\mathrm{a}} \approx 9 \mu_{f}$, shown by the curve next to the rightmost curve $\left(\tan \alpha_{\mathrm{a}} \approx 15 \mu_{f}\right.$ ). Finally, for any given initial state, there exists a terminal tangent velocity. This means that the states of the journal will eventually be described only by the dashed magenta curves for each individual initial state defined by $\bar{V} \cdot \sin \alpha_{\mathrm{a}}=$ const. The faster the state approaches the constant tangent velocity 


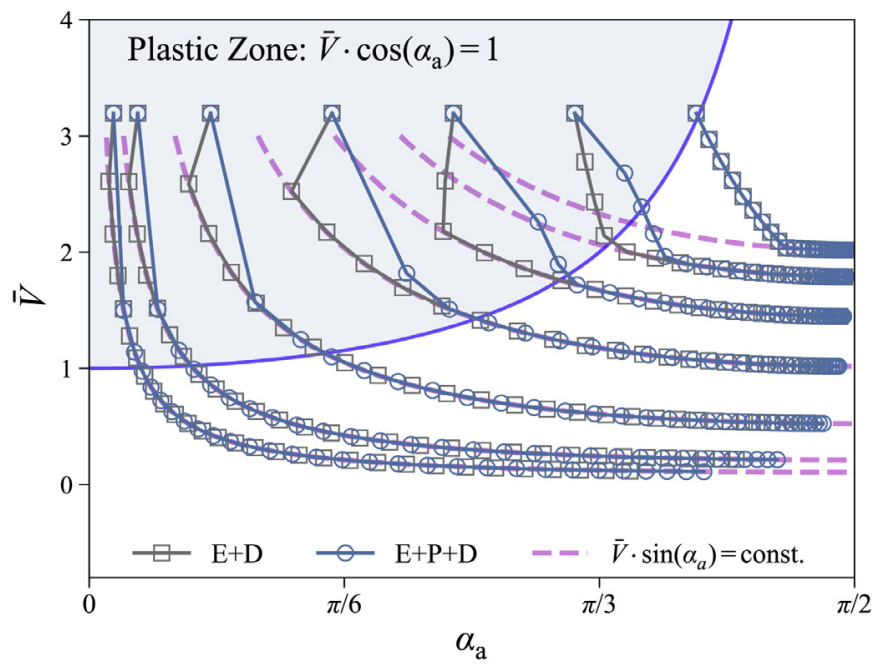

Fig. 16. State portraits of various initial states and models.

line, the faster the tangent velocity stabilizes. Meanwhile, the faster the state approaches the $\alpha_{\mathrm{a}}=\pi / 2$ line, the faster the normal component of velocity stabilizes. Fig. 16 shows the significant difference in state variation between the E+D and $\mathrm{E}+\mathrm{P}+\mathrm{D}$ models, which is especially large for the earlier stage of motion. For the cases initiated in the plastic zone, the $\mathrm{E}+\mathrm{P}+\mathrm{D}$ model is strongly recommended for dynamical analysis and simulation.

\section{Conclusion}

To study the effect of plasticity on dynamic impact in a single journal-bearing planar system, we integrated a new conformal NFD relationship based on the plastic Winkle model into a 2D dynamic solver. The conclusions are as follows:

1. An explicit relation between the critical velocity and payload was obtained (Eq. (16)), which can be used to determine whether the effect of plasticity should be taken into account when simulating a journal-bearing system. The new NFD relationship should be used if the impact velocity exceeds the critical velocity.

2. Compared with damping and frictional dissipation, plastic dissipation is dominant during impact, which results in different dynamic characteristics. In the case when the initial velocity is three-times of $V_{c r}$, the E+P+D model typically required about three impacts to enter the elastic region with all approach angles, while the E+D model required $30 \%$ more impacts (i.e., 4-6 impacts) to enter this region.

3. The travel distance of the journal is much greater in the elastic model compared with the E+P model. In the presence of a lubricating fluid, because the resistance of the fluid is proportional to the travel distance, the unrealistic travel distance calculated by the elastic model may cause large deviations of dynamic characteristics, such as vibration and wear, from the real situation.

Our future work will focus on the effects of plasticity in more complicated real-world cases, in which defects such as misalignment may introduce greater complexity into contact nonlinearity.

\section{Declaration of Competing Interest}

The authors declare that they have no conflict of interest.

\section{Acknowledgment}

This work was supported by the NSFC Basic Science Center Program for "Multiscale Problems in Nonlinear Mechanics" (11988102), by the National Natural Science Foundation of China (11772334 and 11902311), by the Youth Innovation Promotion Association CAS (2018022), and by the Strategic Priority Research Program of the Chinese Academy of Sciences (XDB22040501).

\section{References}

[1] P. Flores, J. Ambrosio, J.C.P. Claro, H.M. Lankarani, C.S. Koshy, A study on dynamics of mechanical systems including joints with clearance and lubrication, Mech. Mach. Theory 41 (3) (2006) 247-261, doi:10.1016/j.mechmachtheory.2005.10.002.

[2] J.F. Rameau, P. Serre, M. Moinet, Clearance vs. tolerance for mobile overconstrained mechanisms, Mech. Mach. Theory 136 (2019) 284-306, doi:10. 1016/j.mechmachtheory.2019.03.007. 
[3] S. Dubowsky, T.N. Gardner, Design and analysis of multi-link flexible mechanisms with multiple clearance connections, J. Eng. Ind. 99 (1) (1977) 88-96, doi:10.1115/1.3439171.

[4] S. Erkaya, Experimental investigation of flexible connection and clearance joint effects on the vibration responses of mechanisms, Mech. Mach. Theory 121 (2018) 515-529, doi:10.1016/j.mechmachtheory.2017.11.014.

[5] R.S. Haines, Survey: 2-dimensional motion and impact at revolute joints, Mech. Mach. Theory 15 (5) (1980) 361-370, doi:10.1016/0094-114X(80) 90013-0.

[6] P. Flores, Kinematics and dynamics of multibody systems with imperfect joints : models and case studies, Lecture Notes in Applied and Computational Mechanics, 34, Springer, Berlin, 2008.

[7] Y. Liu, Y. Yu, A survey of mechanism and robot with clearances, Mech. Sci. Technol. 23 (4) (2004) 454-460.

[8] G. Wang, H. Liu, Research progress of joint effects model in multibody system dynamics, Chin. J. Theor. Appl. Mech. 47 (1) (2015) $31-50$.

[9] Q. Tian, P. Flores, H.M. Lankarani, A comprehensive survey of the analytical, numerical and experimental methodologies for dynamics of multibody mechanical systems with clearance or imperfect joints, Mech. Mach. Theory 122 (2018) 1-57, doi:10.1016/j.mechmachtheory.2017.12.002.

[10] C.S. Liu, K. Zhang, R. Yang, The FEM analysis and approximate model for cylindrical joints with clearances, Mech. Mach. Theory 42 (2) (2007) 183-197, doi: 10.1016/j.mechmachtheory.2006.02.006.

[11] R.S. Haines, A theory of contact loss at resolute joints with clearance, J. Mech. Eng. Sci. 22 (3) (1980) 129-136, doi:10.1243/jmes_jour_1980_022_027_ 02.

[12] P. Ravn, A continuous analysis method for planar multibody systems with joint clearance, Multibody Syst. Dyn. 2 (1) (1998) 1-24, doi:10.1023/a: 1009759826529.

[13] X.P. Wang, W.Z. Lin, X.M. Ji, Z. Gao, X.B. Bai, Y.X. Guo, Dynamic analysis of a planar multibody system with multiple revolute clearance joints, Proc. Inst. Mech. Eng. Part C 233 (10) (2019) 3429-3443, doi:10.1177/0954406218819022.

[14] H.H. Miao, B. Li, J. Liu, A.Q. He, S.K. Zhu, Effects of revolute clearance joint on the dynamic behavior of a planar space arm system, Proc. Inst. Mech. Eng. Part G 233 (5) (2019) 1629-1644, doi:10.1177/0954410018760024.

[15] S. Dubowsky, F. Freudens, Dynamic analysis of mechanical systems with clearances-Part 1. Formation of dynamic model, J. Eng. Ind. 93 (1) (1971) 305, doi:10.1115/1.3427895.

[16] S. Dubowsky, F Freudens, Dynamic analysis of mechanical systems with clearances-Part 2. Dynamic response, J. Eng. Ind. 93 (1) (1971) 310, doi:10. $1115 / 1.3427896$.

[17] A. Persson, On the Stress Distribution of Cylindrical Elastic Bodies in Contact, Chalmers University of Technology, 1964.

[18] M. Ciavarella, P. Decuzzi, The state of stress induced by the plane frictionless cylindrical contact. I. The case of elastic similarity, Int. J. Solids Struct. 38 (26-27) (2001) 4507-4523, doi:10.1016/s0020-7683(00)00289-4.

[19] M. Ciavarella, P. Decuzzi, The state of stress induced by the plane frictionless cylindrical contact. II. The general case (elastic dissimilarity), Int. J. Solids Struct. 38 (26-27) (2001) 4525-4533, doi:10.1016/s0020-7683(00)00290-0.

[20] K.L. Johnson, Contact Mechanics, Cambridge University Press, Cambridge Cambridgeshire; New York, 1985.

[21] W. Goldsmith, Impact: The Theory and Physical Behaviour of Colliding Solids, E. Arnold, London, 1960.

[22] H.M. Lankarani, P.E. Nikravesh, A contact force model with hysteresis damping for impact analysis of multibody systems, J. Mech. Des. 112 (3) (1990) 369-376, doi:10.1115/1.2912617.

[23] E.I. Radzimovsky, Stress Distribution and Strength Condition of Two Rolling Cylinders Pressed Together, University of Illinois Engineering Experiment Station. Bulletin, 1953.

[24] C. Pereira, A. Ramalho, J. Ambrosio, Applicability domain of internal cylindrical contact force models, Mech. Mach. Theory 78 (2014) 141-157, doi:10. 1016/j.mechmachtheory.2014.03.010.

[25] C.M. Pereira, A.L. Ramalho, J.A. Ambrosio, A critical overview of internal and external cylinder contact force models, Nonlinear Dyn. 63 (4) (2011) 681-697, doi:10.1007/s11071-010-9830-3.

[26] C. Pereira, A. Ramalho, J. Ambrosio, An enhanced cylindrical contact force model, Multibody Syst. Dyn. 35 (3) (2015) 277-298, doi:10.1007/ s11044-015-9463-x.

[27] C. Pereira, A. Ramalho, J. Ambrosio, Validation of an enhanced cylindrical contact force model using numerical and analytical approaches, New Trends Mech. Mach. Sci. 24 (2015) 497-504, doi:10.1007/978-3-319-09411-3_53.

[28] C.S. Liu, K. Zhang, L. Yang, Normal force-displacement relationship of spherical joints with clearances, J. Comput. Nonlinear Dyn. 1 (2) (2006) 160-167, doi:10.1115/1.2162872.

[29] X. Fang, C.H. Zhang, X. Chen, Y.S. Wang, Y.Y. Tan, A new universal approximate model for conformal contact and non-conformal contact of spherical surfaces, Acta Mech. 226 (6) (2015) 1657-1672, doi:10.1007/s00707-014-1277-z.

[30] L. Skrinjar, J. Slavic, M. Boltezar, A review of continuous contact-force models in multibody dynamics, Int. J. Mech. Sci. 145 (2018) 171-187, doi:10. 1016/j.ijmecsci.2018.07.010.

[31] G. Wang, C. Liu, Further investigation on improved viscoelastic contact force model extended based on Hertz's law in multibody system, Mech. Mach. Theory 153 (2020) 103986, doi:10.1016/j.mechmachtheory.2020.103986.

[32] J. Zhang, W. Li, L. Zhao, G. He, A continuous contact force model for impact analysis in multibody dynamics, Mech. Mach. Theory 153 (2020) 103946, doi:10.1016/j.mechmachtheory.2020.103946.

[33] R.L. Jackson, I. Green, A finite element study of elasto-plastic hemispherical contact against a rigid flat, J. Tribol. 127 (2) (2005) 343-354, doi:10.1115/ 1.1866166 .

[34] L. Kogut, I. Etsion, Elastic-plastic contact analysis of a sphere and a rigid flat, J. Appl. Mech. 69 (5) (2002) 657-662, doi:10.1115/1.1490373.

[35] L. Kogut, K. Komvopoulos, Analysis of the spherical indentation cycle for elastic-perfectly plastic solids, J. Mater. Res. 19 (12) (2004) 3641-3653, doi:10.1557/Jmr.2004.0468.

[36] N. Ye, K. Komvopoulos, Indentation analysis of elastic-plastic homogeneous and layered media: criteria for determining the real material hardness, J. Tribol. 125 (4) (2003) 685-691, doi:10.1115/1.1572515.

[37] M.R. Brake, An analytical elastic-perfectly plastic contact model, Int. J. Solids Struct. 49 (22) (2012) 3129-3141, doi:10.1016/j.ijsolstr.2012.06.013.

[38] W.J. Stronge, Contact problems for elasto-plastic impact in multi-body systems, Impacts Mech. Syst. 551 (2000) $189-234$.

[39] C. Thornton, Coefficient of restitution for collinear collisions of elastic perfectly plastic spheres, J. Appl. Mech. 64 (2) (1997) 383-386, doi:10.1115/1. 2787319.

[40] H. Ghaednia, D.B. Marghitu, R.L. Jackson, Predicting the permanent deformation after the impact of a rod with a flat surface, J. Tribol. 137 (1) (2015) 8, doi:10.1115/1.4028709.

[41] J. Hu, F. Gao, X. Liu, Y. Wei, An elasto-plastic contact model for conformal contacts between cylinders, Proc. Inst. Mech. Eng. Part J (2019), doi:10.1177/ 1350650119896461.

[42] N. Akhadkar, V. Acary, B. Brogliato, Multibody systems with 3D revolute joints with clearances: an industrial case study with an experimental validation, Multibody Syst. Dyn. 42 (3) (2018) 249-282, doi:10.1007/s11044-017-9584-5.

[43] F. Isaac, F. Marques, N. Dourado, P. Flores, A finite element model of a 3D dry revolute joint incorporated in a multibody dynamic analysis, Multibody Syst. Dyn. 45 (3) (2019) 293-313, doi:10.1007/s11044-018-09659-9.

[44] F. Marques, F. Isaac, N. Dourado, P. Flores, An enhanced formulation to model spatial revolute joints with radial and axial clearances, Mech. Mach. Theory 116 (2017) 123-144, doi:10.1016/j.mechmachtheory.2017.05.020.

[45] J.R. Dormand, P.J. Prince, A family of embedded Runge-Kutta formulae, J. Comput. Appl. Math. 6 (1) (1980) 19-26, doi:10.1016/0771-050X(80)90013-3.

[46] L.F. Shampine, Some practical Runge-Kutta formulas, Math. Comput. 46 (173) (1986) 135-150, doi:10.2307/2008219. 
[47] E. Pennestri, V. Rossi, P. Salvini, P.P. Valentini, Review and comparison of dry friction force models, Nonlinear Dyn. 83 (4) (2016) 1785-1801, doi:10. 1007/s11071-015-2485-3.

[48] Y. Gonthier, J. McPhee, C. Lange, J.C. Piedboeuf, A regularized contact model with asymmetric damping and dwell-time dependent friction, Multibody Syst. Dyn. 11 (3) (2004) 209-233, doi:10.1023/B:MUBO.0000029392.21648.bc.

[49] F. Marques, P. Flores, J.C.P. Claro, H.M. Lankarani, Modeling and analysis of friction including rolling effects in multibody dynamics: a review, Multibody Syst. Dyn. 45 (2) (2019) 223-244, doi:10.1007/s11044-018-09640-6.

[50] M.B. Qian, Z. Qin, S.Z. Yan, L. Zhang, A comprehensive method for the contact detection of a translational clearance joint and dynamic response after its application in a crank-slider mechanism, Mech. Mach. Theory 145 (2020) 19, doi:10.1016/j.mechmachtheory.2019.103717. 